\title{
Efeito do manejo do solo e da matéria orgânica solúvel em água quente na estabilidade de agregados de um latossolo argiloso
}

\author{
Effect of orchard soil management and hot water soluble organic matter on aggregate stability \\ of a clayey oxisol
}

\author{
Carmen Silvia Vieira Janeiro Neves ${ }^{1}$ Christian Feller ${ }^{2}$ Ernest Kouakoua ${ }^{2}$
}

\section{RESUMO}

Estudou-se a estabilidade de agregados de um latossolo vermelho distroférrico submetido às seguintes situações: vegetação nativa de floresta; culturas anuais por 20 anos; pomar cítrico manejado com cobertura verde permanente com a leguminosa Arachis prostrata Bong. ex Benth.; pomar com cobertura de vegetação espontânea (predomínio de gramíneas) controlada com roçadora (3-4 vezes no período de chuvas) e uma gradagem a disco ao ano (no período seco); pomar sem vegetação por meio de capina manual. Os tratamentos no pomar foram mantidos durante 9 anos. A estabilidade de agregados foi determinada em amostras submetidas ou não a tratamento para retirada da matéria orgânica solúvel em água quente. O solo sob vegetação de floresta teve maior quantidade de agregados estáveis, seguido do solo sob pomar com cobertura permanente de leguminosas ou gramíneas. A estabilidade dos agregados não foi afetada pela extração do carbono solúvel em água quente, embora tenha havido correlação positiva entre o teor de carbono solúvel em água quente e a agregação do solo.

Palavras - chave: agregação do solo, estrutura, carbono.

\section{ABSTRACT}

It was studied the aggregate stability of an oxisol maintained with vegetation of native forest; annual crops for 20 years; a citrus orchard mantained during 9 years with permanent ground cover of Arachis prostrata Bong. Ex Benth.; or permanent spontaneous vegetation (mainly gramineous) controlled by mowing (3-4 times in the rain period of rains) and tillage (once a year in the dry period); or bare soil maintained by manual weeding. The aggregate stability was determined in samples submitted or not to treatment to remove hot water soluble organic matter. The forest vegetation provided larger stability followed by the orchard submitted to the permanent covering with leguminous or gramineous plants.
Extraction of hot-water soluble carbon did not cause significant differences in aggregate stability, although there has been a significant correlation between hot water soluble carbon and soil aggregation.

Key words: soil aggregation, structure, carbon.

\section{INTRODUÇÃO}

A agregação é um dos parâmetros que podem ser utilizados para medir a qualidade do solo, pois a manutenção da estrutura do solo facilita a aeração e a infiltração de água e reduz a erodibilidade. A estabilidade dos agregados é influenciada por diversas características do solo, como textura (FELLER et al., 1996), teor de óxidos de ferro e alumínio (OADES \& WATERS, 1991; DUFRANC et al., 2004), teor de matéria orgânica (ROTH et al., 1991; FELLER et al., 1996; BERTOL et al., 2000) e atividade microbiana (TISDALL \& OADES, 1979) e também pelo manejo do solo (CAMBARDELLA\& ELLIOT, 1993; CARPENEDO \& MIELNICZUK, 1990).

No manejo do solo em pomares, o sistema adotado deve proporcionar pouca ou nenhuma competição por água e nutrientes com as árvores e manter o solo protegido, principalmente nos períodos de maior ocorrência de chuvas. De maneira geral, os resultados de trabalhos realizados em pomares cítricos têm demonstrado que a manutenção de vegetação nas entrelinhas da cultura favorece a agregação do solo,

'Departamento de Agronomia, Universidade Estadual de Londrina (UEL), CP 6001, 86051-990, Londrina, PR, Brasil. E-mail: csvjneve@uel.br. Autora para correspondência.

${ }^{2}$ Laboratório MOST, Institut de Recherche pour le Développement (IRD), B.P. 64501, 34394, Montpellier cedex 5, França. Email: feller@mpl.ird.fr. 
como o de CINTRA et al. (1983), na Bahia, que observaram $40 \%$ a mais de agregação em pomar permanentemente conduzido com vegetação nativa ceifada quando comparada aos tratamentos em que a vegetação era eliminada através de gradagem, mesmo que apenas na estação seca. O tipo de vegetação usada na cobertura também tem influência na agregação, como foi observado em um solo franco-argilo-siltoso da Costa do Marfim, em que a estabilidade estrutural foi superior nos tratamentos que receberam leguminosas em relação à vegetação nativa constituída essencialmente de gramíneas (GODEFROY \& BOURDEAUT, 1972).

Alguns trabalhos têm demonstrado existir correlação entre a estabilidade de agregados do solo e o teor de carboidratos solúveis em água quente, que são compostos constituídos principalmente por polissacarídeos originados de exsudatos microbianos (HAYNES \& BEARE, 1997; DEBOSZ et al., 2002). Em trabalhos com rotação a cada 2-5 anos de pastagens e culturas com preparo convencional, em Inceptisols da Nova Zelândia, a estabilidade de agregados aumentou durante a fase de pastagem, seguida de um decréscimo com as culturas, apesar de o teor de matéria orgânica permanecer constante nas duas fases (HAYNES et al., 1991). Concluiu-se que estas mudanças a curto prazo na agregação estariam mais relacionadas a alterações em constituintes orgânicos específicos do que ao teor total de matéria orgânica do solo, pois o teor de carboidratos solúveis em água quente aumentou durante o período de pastagem e diminuiu durante as culturas, com alta correlação com a agregação, enquanto que os índices de correlação com o teor total de $\mathrm{C}$ e $\mathrm{N}$, de carboidratos solúveis em água fria e de ácido-hidrolisáveis foram inferiores (HAYNES \& SWIFT, 1990). Resultados semelhantes foram encontrados também por ANGERS et al. (1993), em um Gleysol do Canadá, e por GIJSMAN \& THOMAS (1995), em um Oxisol da Colômbia. Foi sugerido que o aumento na agregação foi proporcionado pelos carboidratos solúveis em água quente liberados pela biomassa microbiana presente na rizosfera da pastagem (HAYNES \& FRANCIS, 1993).

O presente trabalho teve como objetivo avaliar a estabilidade de agregados em um latossolo vermelho distroférrico submetido a diferentes tipos de usos do solo, antes e depois da extração dos carboidratos solúveis em água quente, relacionando-a com o teor de carbono do solo.

\section{MATERIAL E MÉTODOS}

O clima da região de Londrina (PR), segundo a classificação de Köppen, é do tipo Cfa, subtropical úmido, com temperatura média anual de 20 a $21^{\circ} \mathrm{C}$, com chuvas em todas as estações ( $1.400 \mathrm{a} 1.600 \mathrm{~mm}$ por ano), podendo ocorrer seca no período de inverno. O solo estudado (Latossolo Vermelho distroférrico) está situado no campus da Universidade Estadual de Londrina e, pela análise granulométrica, apresentou $691 \mathrm{~g} \mathrm{~kg}^{-1}$ de argila, $216 \mathrm{~g} \mathrm{~kg}^{-1}$ de silte e $93 \mathrm{~g} \mathrm{~kg}^{-1}$ de areia. A análise química do solo, de acordo com a metodologia descrita em EMBRAPA (1979), realizada por ocasião da instalação do pomar, revelou os seguintes valores para a camada de $0-15 \mathrm{~cm}$ : $\mathrm{pH}\left(\mathrm{H}_{2} \mathrm{O}\right)=5,38$; matéria orgânica $=29,2 \mathrm{~g} \mathrm{dm}^{-3} ; \mathrm{P}\left(\right.$ Mehlish-1) $=2,04 \mathrm{mg} \mathrm{dm}^{-3}$; $\mathrm{K}^{+}=0,2 \mathrm{cmolc} \mathrm{dm}^{-3} ; \mathrm{Ca}^{+2}=4,5 \mathrm{cmolc} \mathrm{dm}^{-3} ; \mathrm{Mg}^{+2}=$ $1,9 \mathrm{cmolc} \mathrm{dm}^{-3} ; \mathrm{H}^{+}+\mathrm{Al}^{3+}=7,0 \mathrm{cmolc} \mathrm{dm}^{-3}$. Para a camada de $15-30 \mathrm{~cm}$, os resultados foram: $\mathrm{pH}\left(\mathrm{H}_{2} \mathrm{O}\right)=5,02$; matéria orgânica $=17,5 \mathrm{~g} \mathrm{dm}^{-3} ; \mathrm{P}($ Mehlish-1 $)=0,92 \mathrm{mg}$ $\mathrm{dm}^{-3} ; \mathrm{K}^{+}=0,1 \mathrm{cmolc} \mathrm{dm}^{-3} ; \mathrm{Ca}^{+2}=3,3 \mathrm{cmolc} \mathrm{dm}^{-3} ; \mathrm{Mg}^{+2}$ $=3,2 \mathrm{cmolc} \mathrm{dm}-3 ; \mathrm{H}^{+}+\mathrm{Al}^{3+}=0,6 \mathrm{cmolc} \mathrm{dm}{ }^{-3}$.

A área foi mantida com um pomar de citros, conduzido durante 9 anos com um experimento de manejo de solo nas entrelinhas (NEVES et al., 1998; NEVES et al., 2005). Todos os tratamentos receberam iguais quantidades de calcário dolomítico a cada 3-4 anos e adubação mineral anual (sulfato de amônio, superfosfato simples e cloreto de potássio) com base nos teores dos elementos no solo e nas produções médias anuais. Foram estudados os seguintes sistemas de manejo: cobertura verde durante todo o ano com a leguminosa perene Arachis prostrata Benth.; cobertura de vegetação espontânea, com predomínio de gramíneas perenes controladas pelo uso de uma gradagem a disco anualmente (no período seco) e de roçadora (três a quatro vezes no período de chuvas); solo mantido descoberto por meio de capina manual o ano inteiro. Foram retiradas também amostras do mesmo tipo de solo de áreas localizadas ao lado do pomar sob floresta nativa (tropical subperenifólia) e cultivada por 20 anos, em sistema de preparo de solo convencional, com culturas anuais (no ano da avaliação, as culturas foram aveia no inverno e algodão no verão). As amostras foram compostas de seis subamostras, com quatro repetições, em duas profundidades (0-10 e 10$20 \mathrm{~cm})$.

A estabilidade dos agregados foi determinada de acordo com o método de KEMPER \& ROSENAU (1986), passando-se a amostra por peneiramento a $2 \mathrm{~mm}$ (KOUAKOUA et al., 1997). As amostras foram imersas em água fria (30 minutos) ou quente $\left(60\right.$ minutos a estufa a $\left.90^{\circ} \mathrm{C}\right)$ e depois peneiradas (malha $200 \mu \mathrm{m}$ ) em um agitador vertical (KEMPER \& ROSENAU, 1986), com 1,3cm de amplitude vertical e freqüência 35 ciclos minuto $^{-1}$, durante 6 minutos. A fração que passou pela peneira teve o teor de argila + 
silte fino determinado por meio do método da pipeta (ROBINSON, 1967). A fração restante na peneira, representando a fração $\mathrm{AE}+\mathrm{AG}$ (agregados estáveis mais areia grossa), teve a massa determinada e foi dispersa com $\mathrm{NaOH} 0,05 \mathrm{M}$ e peneirada novamente para obtenção da fração $\mathrm{AG}$ (que não passou pela peneira depois da desagregação) e AE. A taxa de agregados estáveis (AE) expressa em $\mathrm{g} \mathrm{kg}^{-1}$ foi calculada pela fórmula $\mathrm{AE}=[(\mathrm{AE}+\mathrm{AG})-\mathrm{AG})]$.

A dosagem do carbono solubilizado pela água quente foi feita no filtrado obtido por centrifugação (4.000rpm durante 30 minutos). O teor de carbono solúvel foi avaliado por um analisador Shimadzu TOC 5000. O teor de carbono total do solo foi determinado por combustão via seca em um analisador CHN 600, Leco Corporation. Todas as determinações foram realizadas no Laboratório de Solos Cultivados do IRD, em Montpellier, França.

Os resultados foram analisados em delineamento inteiramente ao acaso, com quatro repetições. Os tratamentos foram dispostos num esquema fatorial, sendo os fatores uso da terra e temperatura da água utilizada na avaliação da agregação (quente ou fria).

\section{RESULTADOS E DISCUSSÃO}

O solo submetido à vegetação de floresta nativa foi o que apresentou a maior estabilidade de agregados, superior a todos os demais, nas duas profundidades (Tabela 1). Os menores teores de agregados estáveis foram observados no tratamento de culturas anuais por 20 anos e no pomar capinado. Houve um aumento significativo da porcentagem de agregados estáveis quando o pomar foi submetido a cobertura vegetal (leguminosa A. prostrata ou gramíneas), comparando-se com o pomar capinado, que, por ter sido mantido descoberto, provavelmente foi desagregado pelo impacto das gotas de chuva.

Comparando-se os dois tratamentos de cobertura vegetal do pomar, a estabilidade de agregados foi semelhante nos dois sistemas, indicando que estes dois sistemas de manejo contribuíram de forma semelhante para a manutenção da agregação do solo, mesmo considerando que o tratamento vegetação espontânea de gramíneas recebeu uma gradagem ao ano, o que poderia diminuir a agregação. As raízes das gramíneas são decompostas mais lentamente (TISDALL \& OADES, 1982; FAHAD et al., 1982) e possuem uma importante população de fungos micorrízicos (TISDALL \& OADES, 1979) atuando como importantes agentes estabilizadores dos
Tabela 1 - Agregados estáveis em solo submetido às condições de cultura anual, mata e de pomar manejado com Arachis prostata, roçadeira/grade ou capina, na profundidade de $0-10$ e $10-20 \mathrm{~cm}$, submetidos a tratamento com água fria ou quente.

\begin{tabular}{|c|c|c|}
\hline \multirow[b]{2}{*}{ Tratamento } & \multicolumn{2}{|c|}{ Agregados estáveis $\left(\mathrm{g} \mathrm{kg}^{-1}\right)$} \\
\hline & Água fria & Água quente \\
\hline Profundidade & \multicolumn{2}{|c|}{$0-10 \mathrm{~cm}$} \\
\hline Cultura anual & $550,6 \mathrm{~A} \mathrm{c}^{*}$ & $548,2 \mathrm{~A} \mathrm{~d}$ \\
\hline Floresta nativa & $751,9 \mathrm{~A} \mathrm{a}$ & $792,5 \mathrm{~A}$ a \\
\hline Pomar - Arachis prostrata & $638,6 \mathrm{~A} \mathrm{~b}$ & $663,3 \mathrm{~A} \mathrm{~b}$ \\
\hline Pomar - veget.espontânea & $626,0 \mathrm{~A} \mathrm{~b}$ & $623,3 \mathrm{~A} \mathrm{bc}$ \\
\hline Pomar - capina & $552,0 \mathrm{~A} \mathrm{c}$ & $567,5 \mathrm{~A} \mathrm{~cd}$ \\
\hline DMS (tratam.) & \multicolumn{2}{|l|}{72,25} \\
\hline DMS (temp. água) & \multicolumn{2}{|l|}{50,93} \\
\hline CV $(\%)$ & \multicolumn{2}{|l|}{5,582} \\
\hline Profundidade & \multicolumn{2}{|c|}{$10-20 \mathrm{~cm}$} \\
\hline \multicolumn{3}{|l|}{ Tratamento } \\
\hline Cultura anual & $548,3 \mathrm{~A} \mathrm{c}^{*}$ & $557,6 \mathrm{~A} \mathrm{bc}$ \\
\hline Floresta nativa & $688,5 \mathrm{~A} \mathrm{a}$ & $726,1 \mathrm{~A} \mathrm{a}$ \\
\hline Pomar - Arachis prostrata & $568,5 \mathrm{~A} \mathrm{bc}$ & $591,7 \mathrm{~A} \mathrm{~b}$ \\
\hline Pomar - veget.espontânea & $629,0 \mathrm{~A} \mathrm{ab}$ & $585,5 \mathrm{~A} \mathrm{~b}$ \\
\hline Pomar - capina & $545,7 \mathrm{~A} \mathrm{c}$ & $488,0 \mathrm{~A} \mathrm{c}$ \\
\hline DMS (tratam.) & 74,74 & \\
\hline DMS (temp. água) & 52,68 & \\
\hline CV $(\%)$ & 6,149 & \\
\hline
\end{tabular}

*Médias seguidas da mesma letra, minúscula na vertical e maiúscula na horizontal, não diferem entre si pelo teste de Tuckey a $5 \%$ de probabilidade de erro.

macroagregados. Na Austrália, em pomar cítrico, GREACEN \& PERKMAN (1953) tiveram redução do estado de agregação do solo com leguminosa como adubo verde, que teve $1,93 \%$ de agregação, enquanto a vegetação natural ceifada teve $20,22 \%$ e o tratamento herbicida teve 7,20\% de agregação. Este dado deve-se provavelmente ao fato de ter sido o adubo verde anual e não perene, sendo semeado após um cultivo com arado, pois, no mesmo trabalho, o teor de carbono não apresentou diferenças, depois de cinco anos de experimento.

Verificou-se correlação positiva entre o teor de carbono total do solo e os agregados estáveis (Figura 1), confirmando o observado em outros trabalhos sobre esta importante função da matéria orgânica na manutenção da estrutura do solo (TISDALL \& OADES, 1979; SIX et al., 1998; BERTOL et al., 2000). Também se observou correlação entre o teor de carbono solúvel em água quente e a estabilidade de agregados (Figura 2), corroborando os resultados 


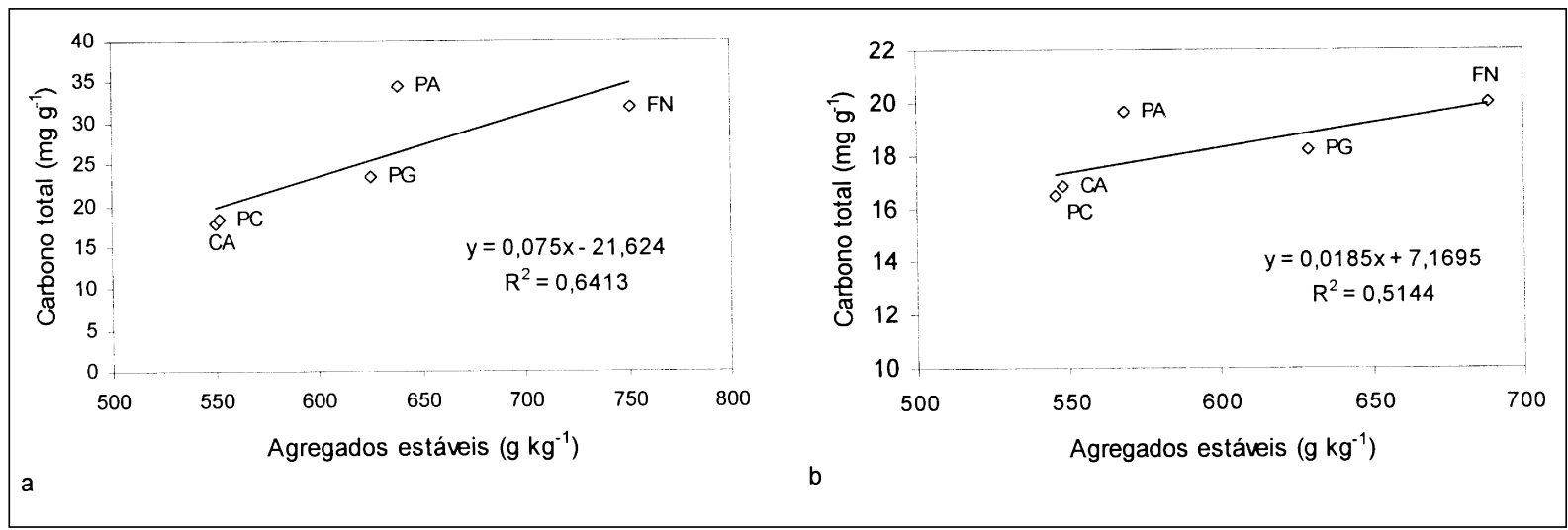

Figura 1 - Relação entre o teor de carbono total do solo e os agregados estáveis (determinados em água fria) para as situações estudadas, nas profundidades de 0-10 (a) e 10-20 cm (b).

CA: cultura anual; PC: pomar capinado; PG: pomar com gramíneas; PA: pomar com Arachis prostrata; FN: floresta nativa.

encontrados por GIJSMAN \& THOMAS (1995) e HAYNES \& SWIFT (1990).

Analisando-se os coeficientes de correlação entre os agregados estáveis e o carbono total e solúvel em água quente (Figuras 1 e 2), seria possível supor que o carbono solúvel é um dos componentes da matéria orgânica responsáveis pela manutenção da estrutura do solo. Porém, ao se comparar os resultados da análise de agregados realizada com e sem o tratamento com água quente (Tabela 1), verificou-se que a agregação não foi reduzida pela extração da porção da matéria orgânica solúvel em água quente. Isto pode estar relacionado ao fato de que a agregação está relacionada também a outros fatores, como a presença de óxidos de ferro e alumínio nos Oxisolos, como é o caso do solo estudado, e que estão presentes em menores quantidades em outros tipos de solos, bem como à porção mais estável da matéria orgânica do solo (OADES \& WATER, 1991;
SHEPHERD et al., 2001). FELLER et al. (1996) observaram diferenças entre tipos de solo, principalmente para os microagregados $(0-5 \mu \mathrm{m})$, que, nos solos oxídicos, apresentaram alta estabilidade para qualquer nível de matéria orgânica, enquanto que, nos Vertisols, estes agregados apresentaram a estabilidade variável com a matéria orgânica do solo.

Em oxisolos do Congo, KOUAKOUA et al. (1997) também não encontraram diferenças ao realizarem a determinação dos agregados estáveis antes e depois da extração com água quente, apesar de existir alta correlação entre a estabilidade e o teor de carbono solúvel em água quente, o que indica que outros atributos do solo participam da formação e estabilização de agregados. Segundo os autores, a medição do efeito direto do teor das frações orgânicas sobre a agregação é importante para testar as conclusões que são tiradas a partir de dados estatísticos de correlações entre os atributos do solo.

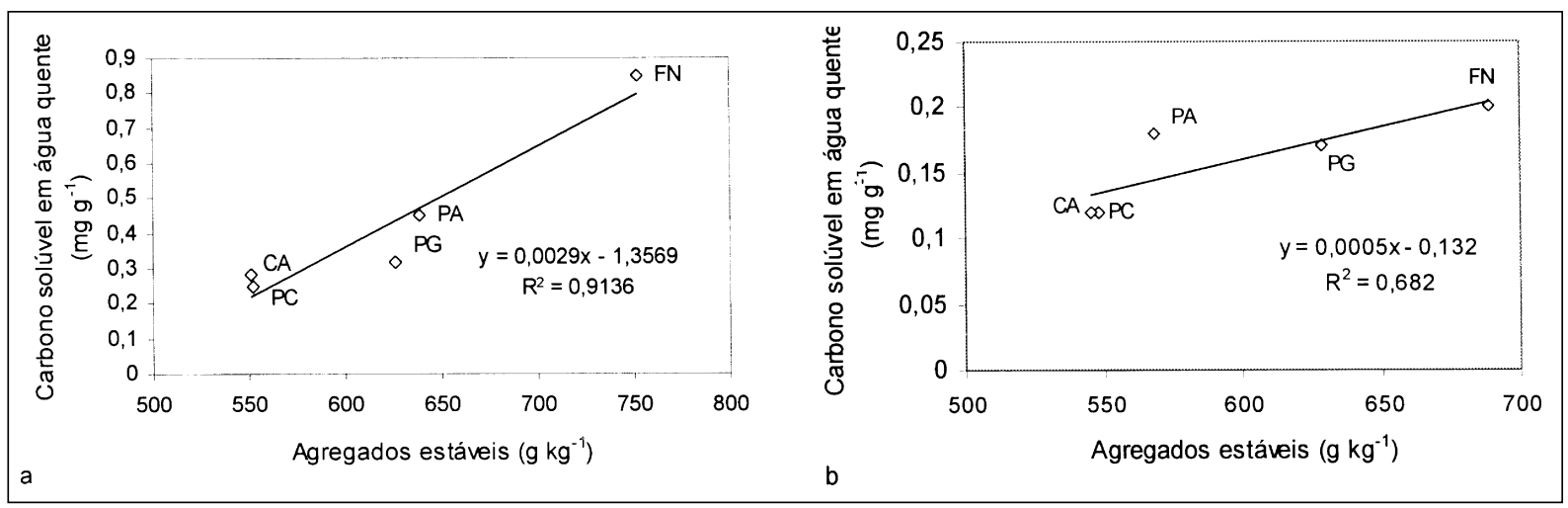

Figura 2 - Relação entre o teor de carbono do solo solúvel em água quente e os agregados estáveis (determinados em água fria) para as situações estudadas, nas profundidades de 0-10 (a) e 10-20 cm (b)

CA: cultura anual; PC: pomar capinado; PG: pomar com gramíneas; PA: pomar com Arachis prostrata; FN: floresta nativa.

Ciência Rural, v.36, n.5, set-out, 2006. 


\section{CONCLUSÕES}

A estabilidade de agregados foi reduzida quando o solo passou da vegetação de floresta para culturas. Esta redução foi mais acentuada para o solo com culturas anuais e com pomar capinado e menor para o solo do pomar com cobertura vegetal de gramíneas ou leguminosas. Embora tenha sido verificada a correlação entre a estabilidade de agregados e o teor de carbono solúvel em água quente, a estabilidade não foi alterada pela extração do carbono solúvel em água quente.

\section{AGRADECIMENTOS}

Os autores agradecem à Coordenação de Aperfeiçoamento de Pessoal de Nível Superior, Ministério da Educação (CAPES/MEC), pela oportunidade de realização do trabalho por meio de Bolsa Doutorado Sanduíche e ao Conselho Nacional de Desenvolvimento Científico e Tecnológico (CNPq), pela concessão de bolsa de Produtividade em Pesquisa ao primeiro autor.

\section{REFERÊNCIAS}

ANGERS, D.A. et al. Early changes in water-stable aggregation induced by rotation and tillage in a soil under barley production. Canadian Journal of Soil Science, v.73, n.1, p.51-59, 1993.

BERTOL, I. et al. Propriedades físicas do solo relacionadas a diferentes níveis de oferta de forragem de capim-elefante-anão cv. Mott. Pesquisa Agropecuária Brasileira, v.35, n.5, p.1047-1054, 2000.

CAMBARDELlA, C.A.; ELLIOT, E.T. Carbon and nitrogen distribution in aggregates from cultivated and native grassland soils. Soil Science Society of America Journal, v. 57, n.4, p.1071-1076, 1993

CARPENEDO, V.; MIELNICZUK, J. Estado de agregação e qualidade de agregados de latossolos roxos, submetidos a diferentes sistemas de manejo. Revista Brasileira de Ciência do Solo, v.14, n.1, p.99-105, 1990.

CINTRA, F.L.D. et al. Caracterização física do solo submetido a práticas de manejo em pomar de Laranja "Baianinha". Pesquisa Agropecuária Brasileira, v.18, n.2, p.173-179, 1983.

DUFRANC, G. et al. Atributos físicos, químicos e biológicos relacionados com a estabilidade de agregados de dois latossolos em plantio direto no Estado de São Paulo. Revista Brasileira de Ciência do Solo, v.28, n.3, p.505$517,2004$.

EMBRAPA - EMPRESA BRASILEIRA DE PESQUISA AGROPECUÁRIA. Manual de métodos de análise de solo. Rio de Janeiro: SNLCS, 1979. n.p.

FAHAD, A.A. et al. Soil physical properties as affected by soybean and other cropping sequences. Soil Science Society of America Journal, v.46, n.2, p.377-381, 1982.
FELLER, C. et al. Aggregation and organic matter storage in kaolinitic and smectitic tropical soils. In: CARTER, M.R.; STEWART, B.A. (Ed). Structure and organic matter storage in agricultural soils. Boca Raton: Lewis, 1996. p. 309-359.

DEBOSZ, K. et al. Carbohydrates in hot water extracts of soil aggregates as influenced by long-term management. Communications in Soil Science and Plant Analysis, v.33, n.3/4, p.623-634, 2002.

GIJSMAN, A.J.; THOMAS, R.J. Aggregate size distribution and stability of an oxisol under legume-based and pure grass pastures in the eastern colombian savannas. Australian Journal of Soil Research, v.33, n.1, p.153-165, 1995.

GODEFROY, J.; BOURDEAUT, T. Action des plantes de couverture sur les caractéristiques chimiques, biologiques et structurales d'un sol de verger de Côte d'Ivoire. Fruits, v.27, n.5, p.349-353, 1972 .

GREACEN, E.L.; PERKMAN, O. Soil-structure changes in a long-term citrus experiment. Australian Journal of Agricultural Research, v.4, n.2, p.193-203, 1953.

HAYNES, R.J.; FRANCIS, G.S. Changes in microbial biomass $\mathrm{C}$, soil carbohydrate composition and aggregate stability induced by growth of selected crop and forage species under field conditions. Journal of Soil Science, v.44, p.665-675, 1993.

HAYNES, R.J.; BEARE, M.H. Influence of six crop species on aggregate stability and some labile organic matter fractions. Soil Biology and Biochemistry, v.29, p.1647-1653, 1997.

HAYNES, R.J.; SWIFT, R.S. Stability of soil aggregates in relation to organic constituents and soil water content. Journal of Soil Science, v.41, p.73-83, 1990.

HAYNES, R.J. et al. Influence of mixed cropping rotations (pasture-arable) on organic matter content, water stable aggregation and clod porosity in a group of soils. Soil \& Tillage Research, v.19, p.77-87, 1991.

KEMPER, W.D.; ROSENAU, R.C. Aggregate stability and size distribution. In: KLUTE, A. Methods of soil analysis. 2.ed. Madison: American Society of Agronomy, 1986. pt 1: Physical and mineralogical methods, p.425-443.

KOUAKOUA, E. et al. La matière organique soluble à l'eau chaude et la stabilité de l'agregation. Aspects méthodologiques et application à des sols ferralitiques du Congo. European Journal of Soil Science, v.48, p.239-247, 1997.

NEVES, C.S.V.J. et al. Matéria orgânica das frações de um latossolo vermelho distroférrico sob diferentes sistemas de uso e manejo. Semina, Londrina, v.26, n.1, p.17-26, 2005.

NEVES, C.S.V.J. et al. Influência de sistemas de manejo de solo em pomar de tangerina poncã sobre limão cravo em um latossolo roxo. Revista Brasileira de Fruticultura, Cruz das Almas, v.20, n.3, p.367-374, 1998.

OADES, J.M.; WATERS, A.G. Aggregate hierarchy in soils. Australian Journal of Soil Research, v.29, n.6, p.815828,1991 .

Ciência Rural, v.36, n.5, set-out, 2006. 
ROBINSON, J.B.D. Soil particle size fractions and nitrogen mineralization. Journal of Soil Science, v.18, p.109-117, 1967.

ROTH, C.H. et al. Análise de fatores físicos e químicos relacionados com a agregação de um latossolo roxo distrófico. Revista Brasileira de Ciência do Solo, v.15, n.3, p.241248, 1991.

SHEPHERD, T.G. et al. Tillage-induced changes to soil structure and organic carbon fractions in New Zealand soils. Australian Journal of Soil Research, v.39, n.3, p.465-489, 2001.
SIX, J. et al. Aggregation and soil organic matter accumulation in cultivated and native grassland soils. Soil Science Society of America Journal, v.62, p.1367-1377, 1998.

TISDALL, J.M.; OADES, J.M. Organic matter and waterstable aggregates in soil. Journal of Soil Science, v.33, p.141-163, 1982.

TISDALL, J.M.; OADES, J.M. Stabilization of soil aggregates by the root systems of ryegrass. Australian Journal of Soil Research, v.17, n.3, p.429-441, 1979. 